\title{
Anti-biogram pattern of uro-pathogens isolated from patients in a Tertiary Care Hospital in Karnataka, India
}

\author{
Charu Kaushik ${ }^{1}$, Namratha Kurunji Gangadhar ${ }^{2, *}$, Subrahmanya Bhat K. ${ }^{3}$, Subbannayya Kotigadde ${ }^{4}$ \\ ${ }^{1}$ PG Student, ${ }^{2,3}$ Assistant professor, ${ }^{4}$ Professor, Dept. of Microbiology, K.V.G. Medical College and Hospital, Kurunjibag Sullia, \\ Karnataka, India
}

*Corresponding author:

Email: drnamrathak@gmail.com

\begin{abstract}
Introduction: Urinary tract infection (UTI) is one of the important causes of morbidity in the community.Development of antibiotic resistance among uro-pathogensposeproblem in the treatment of UTI.Hence it is essential to examine the prevalence of such uro-pathogens and study theiranti-biogram.

Materials and Methods: Bacteria isolated from midstream urine samples collected aseptically from 266 patients over a period of 6 months from November 2016 to April 2017 were identifiedbystandard microbiological procedures. Anti-biogram was done by Kirby-Bauer disc diffusion method and interpreted as per the CLSI guidelines.

Result: Out of 266 urine samples, 136 (51.12\%) were found to be positive for microbial isolates, of which $96(71.3 \%)$ samples were from females and $40(29.4 \%)$ samples were from males. Escherichia coli was the predominant organism (47.05\%) followed by Pseudomonas species (13.2\%), Enterococcus species (11.7\%), Klebsiella species (7.3\%), Citrobacter koseri (4.4\%) and Proteus species $(2.9 \%)$. Enterobacteriaceae shows highest sensitivity towards nitrofurantoin (86\%), amikacin (68\%) and gentamicin (59\%) and resistance towards norfloxacin (74\%). Sensitivity of Pseudomonas spp was $72.2 \%$ for piperacillin/tazobactam, $44.4 \%$ for amikacin and $66 \%$ resistance to ceftazidime. Sensitivity pattern of Enterococci spp to nitrofurantoin $96.6 \%$, aminoglycosides $78 \%$, and fluroquinolones $50 \%$.

An attempt was made to study ESBL production among Enterobacteriaceae members. The isolates were $54.4 \%$ multidrug resistant (MDR) and 38.8\% were ESBL producers.

Conclusion: In our study E.coli was the most common uropathogen isolated. Patterns of antibiotic resistance in a wide variety of pathogenic organism were noted. To prevent the development of resistance, periodic evaluation of antibiotic susceptibility patterns is necessary.
\end{abstract}

Keywords: Enterobacteriaceae, Extended spectrum beta-lactamase, Uro-pathogens.

\section{Introduction}

Urinary tract infections (UTI) are a common, yet a major health problem in all ages accounting for high morbidity and financial cost. ${ }^{1}$ About 150 million people develop UTI each year globally. It accounts for nearly 7 million office visits and 1 million emergency department visits, resulting in about 1 million hospitalizations. $^{2}$

Urinary tract infection actually refers to any type of urothelial inflammatory response resulting from bacterial invasion of the urinary tract. It is commonly following procedures like catheterization and cystoscopy, making it the most common nosocomial infection, accounting for upto $40 \%$ of all nosocomial infections. ${ }^{3}$ It is also commonly seen in patients with diabetes and other diseases which suppress the immune system and also in those with structural and neurological abnormalities which interfere with urinary out flow. Although UTI is seen in both genders, studies suggest a higher prevalence in women than in men. ${ }^{4}$ Nearly 1in 3 women have atleast 1 episode of UTI requiring antimicrobial therapy by the age of 24 years. Almost half of all women experience 1 UTI during life time. ${ }^{5}$ In elderly men, an enlarged prostate can hinder the flow of urine, thus increasing the risk of infection. Renal calculi can also be a risk factor in both the gender. The manifestations may vary from mild asymptomatic cystitis to pyelonephritis and septicemia. ${ }^{6}$

Even though, the microbiology of UTI has been studied for many years, there has been a subsequent change in the characteristics of the micro-organisms particularly due to antimicrobial resistance. ${ }^{6}$ This increasing antimicrobial resistance complicates uncomplicated UTI treatment by increasing patient morbidity, cost of reassessment, retreatment and use of broader spectrum of antibiotics. In most cases of UTI, an empirical treatment is initially given before results of urine culture and sensitivity are available. But the increasing problem of antibiotic resistance necessitates a periodic evaluation of antimicrobial activity to update the information. ${ }^{7}$

Thus, this study was carried out to determine the prevalent uropathogens in our area and antibiotic resistance patterns among them.

\section{Material and Methods}

Study site and duration: The present study was retrospective, hospital based and carried out in the Department of Microbiology KVG Medical College and Hospital, Sullia from November 2016 to April 2017.

Inclusion criteria: All suspected cases of UTI 
Exclusion criteria: Patients on antibiotics in prior week.

Collection and processing of samples: Mid-stream clean catch urine samples were collected from all patients suspected of UTI and transported immediately to the laboratory without any delay. Microscopic examination (wet mount) of urine was done. The wet preparation technique is a quick and simple method ideal for the identification of crystals, casts and pus cells.

Urine culture was done on blood agar, MacConkey's agar and cysteine lactose electrolyte deficient (CLED) agar by semi-quantitative method. Significant growth was considered in case of colony count amounting to $\geq 10^{5}$ colony forming unit $/ \mathrm{mL}$ (CFU/mL) as per standard. Symptomatic cases with a lower count were also considered for the study. Repeated isolation of a single pathogen in freshly collected MSU or in symptomatic cases were considered significant even with a low bacterial count.

Identification of bacteria was done by using standard microbiological procedures. ${ }^{8,9}$ Candida species were identified by Gram's staining, germ tube formation ${ }^{10}$ and by using Chrome agar.

Antimicrobial susceptibility testing: This was done by the modified Kirby Bauer disc diffusion method according to the Clinical Laboratory Standards Institute (CLSI) guidelines. ${ }^{11}$ All Enterobacteriaceae members were tested against nitrofurantoin $(300 \mu \mathrm{g})$, amikacin $(30 \mu \mathrm{g})$ gentamicin $(10 \mu \mathrm{g})$, cefotaxime $(30 \mu \mathrm{g})$, norfloxacin $(10 \mu \mathrm{g})$, nalidixic acid $(30 \mu \mathrm{g})$, ceftriaxone $(30 \mu \mathrm{g})$, ceftazidime $(30 \mu \mathrm{g})$. Pseudomonas aeruginosa were tested against ceftazidime $(30 \mu \mathrm{g})$ sparfloxacin (30ug), ciprofloxacin $(5 \mu \mathrm{g}), \quad$ cotrimoxazole $(1.2523 .75 \mu \mathrm{g})$, ceftriaxone $(30 \mu \mathrm{g})$, amikacin $(30 \mu \mathrm{g})$, gentamicin $(10 \mu \mathrm{g})$, piperacillin/tazobactam $(100 / 10 \mu \mathrm{g})$, imipenem $(10 \mu \mathrm{g})$, meropenem $(10 \mu \mathrm{g})$, and ceftriaxone/tazobactam $(30 / 10 \mu \mathrm{g})$. Enterococcus spp. were tested against nitrofurantoin $(300 \mu \mathrm{g})$, gatifloxacin
$(5 \mu \mathrm{g})$, norfloxacin $(10 \mu \mathrm{g})$, nalidixic acid $(30 \mu \mathrm{g})$, Amikacin $(30 \mu \mathrm{g})$ high level gentamicin $(120 \mu \mathrm{g})$.

E.coli ATCC 25922, and P.aeruginosa ATCC 27853 were used as controls.

\section{ESBL detection}

All the isolates showing resistance to third generation cephalosporins, namely ceftazidime, ceftriaxone and cefotaxime, were further tested for confirmation of extended spectrum $\beta$-lactamase production by phenotypic methods.

\section{Phenotypic confirmatory test for extended-spectrum beta-lactamase production}

As per the guidelines of CLSI, the test was done with confluent growth of the test isolates $(0.5$ McFarland standard) on Mueller-Hinton agar plates, with a distance of $20 \mathrm{~mm}$ of disc containing cefotaxime disc $(30 \mu \mathrm{g} / \mathrm{disc})$ and ceftazidime disc $(30 \mu \mathrm{g} / \mathrm{disc})$ with the combination of cefotaxime with clavulanate $(30 \mu \mathrm{g}$ $+10 / \mu \mathrm{g} / \mathrm{disc})$ and ceftazidime with clavulanate $(30 \mu \mathrm{g}+$ $10 / \mu \mathrm{g} / \mathrm{disc}$ ). Plates were incubated overnight at $37^{\circ} \mathrm{C}$, and after incubation a zone of inhibition that showed $\geq 5$ $\mathrm{mm}$ increased in the cephalosporin discs and their respective cephalosporin/clavulanate discs was taken to be a phenotypic confirmation of ESBL production. ${ }^{12}$

\section{Results}

Out of 266 urine samples, 136(51.12\%) were found to be positive for microbial isolates. Overall prevalence of UTI was higher in female $96(71.3 \%)$ and prevalence among males was 40(29.4\%). Significant bacteriurea was seen in $136(51.1 \%)$ patients and $13(4.8 \%)$ patients had an insignificant colony count. No growth was seen in $100(37.5 \%)$ specimen and mixed growth was seen in $17(6.3 \%)$ samples. Department wise distribution showed that maximum numbers of samples were from obstetrics \& gynecology (40\%) followed by surgery $(22 \%)$, medicine $(15 \%)$, pediatrics $(9 \%)$, Orthopedics (8\%) and ENT\& Dermatology (3\%).

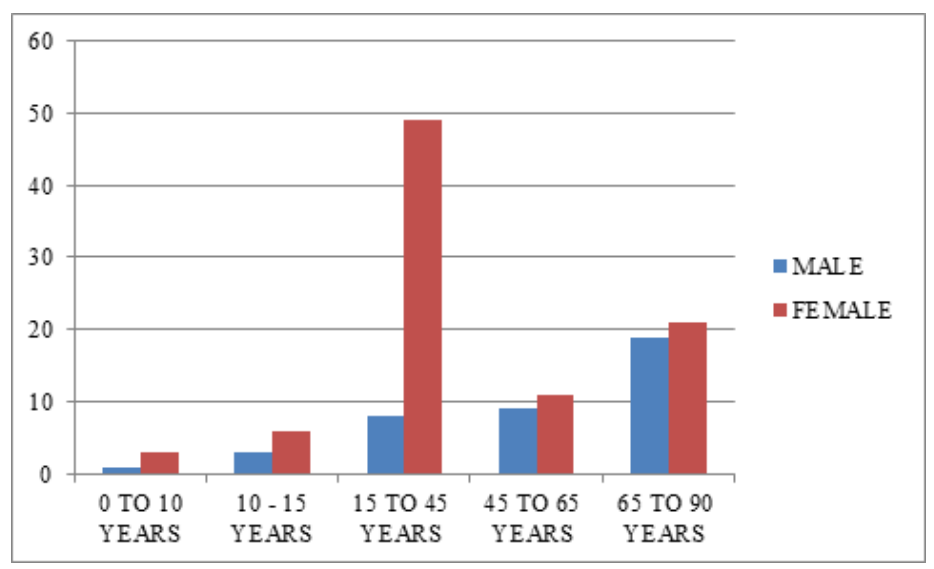

Fig. 1: Age/Sex wise distribution of patients with UTI 
Most of the cases were recorded in 15-49 (41.9\%) years of age group followed by 65-90(29.4\%) years. In both age groups females showed higher risk of developing UTI than male. Age/sex wise distribution is given in (Fig. 1).

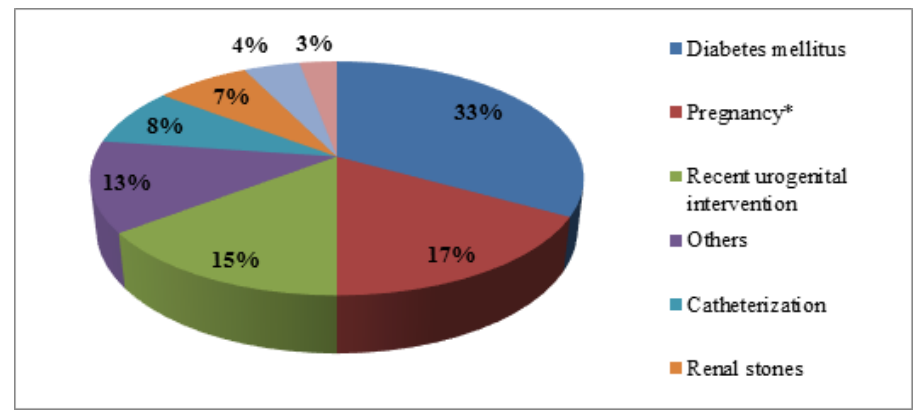

\section{Fig. 2: Risk factors in subjects with UTI}

*23.9\% females with UTI were pregnant.

Diabetes $(33.08 \%)$ was the most common factor associated with UTI in our study. Pregnancy (16.9\%) was the second most common risk factor associated with UTI. While the recent history of uro-genital instrumentation other than catheterization was present in $14.7 \%$ of the study subjects, catheterization alone posed a significant risk factor seen in $8.08 \%$ of the patients.Others $(12.5 \%)$ include patients in reproductive age group without any othercomplication. (Fig. 2)

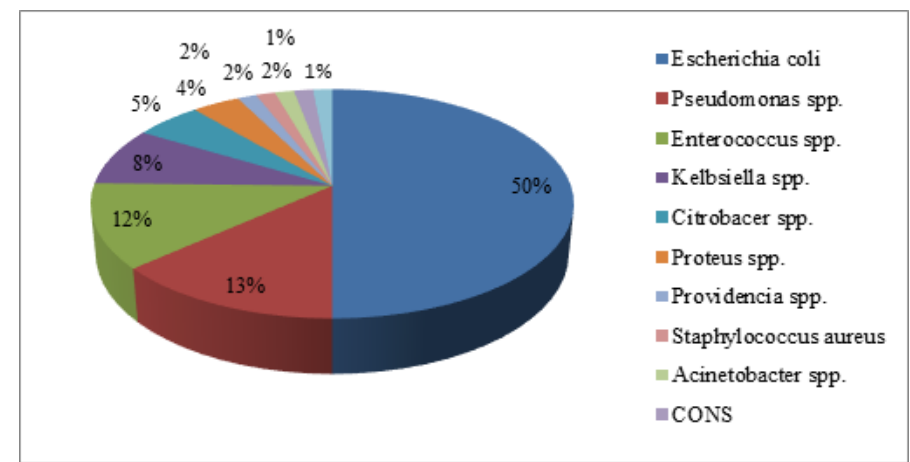

Fig. 3: Frequency of various pathogenic organisms in the study $(n=136)$

Escherichia coli were the predominant organism $(47.05 \%)$ and second most common organism isolated were Pseudomonas species $(13.2 \%)$ and third most common organism was Enterococci species (11.7\%) followed by Klebsiella species(7.3\%), Citrobacter koseri (4.4\%), Proteus species (2.9\%). Incidence of Providencia spp, Staphylococcus aureus, Acinetobacter spp, Coagulase negative Staphylococcus (CONS) and Candida species were (1.4\%). (Fig. 3)

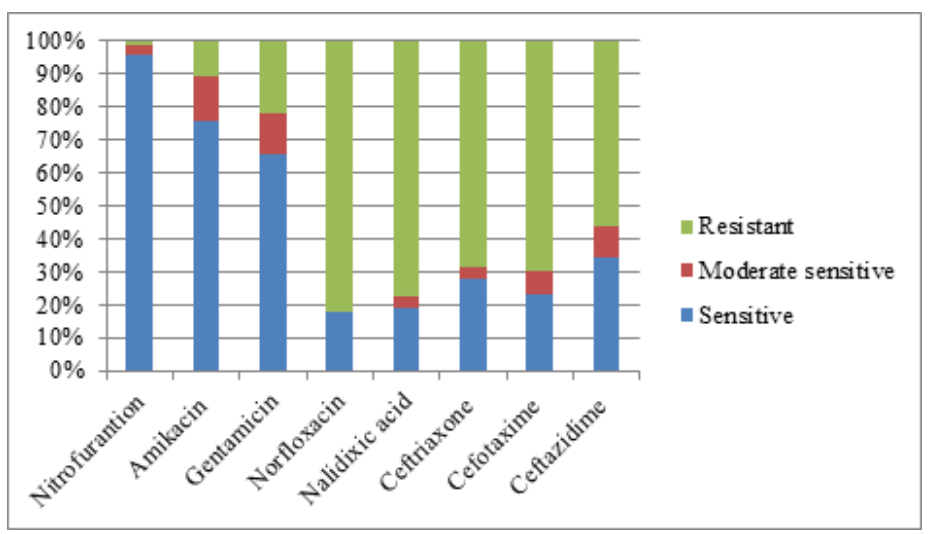

Fig. 4: Antibiotic sensitivity pattern for members of Enterobacteriaceae (n=92) 
A total of 92 Enterobacteriaceae members were isolated from the urine samples. They showed maximum sensitivity towards nitrofurantion (95.5\%), amikacin (68\%) and gentamicin (59\%) and maximum resistance towards norfloxacin $(82.6 \%)$, nalidixic acid $(70 \%)$ and third generation cephalosporin (56-63\%). Antibiotic sensitivity pattern is given in Fig. 4.

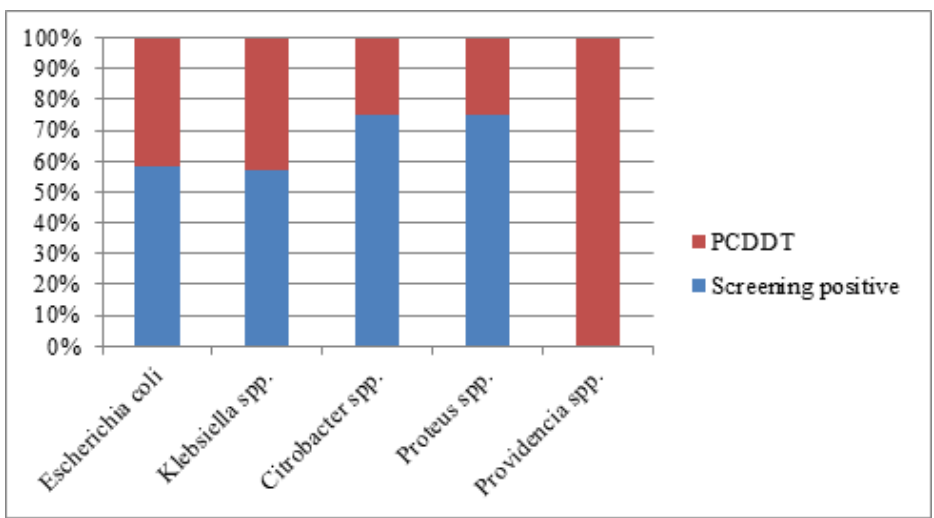

Fig. 5: Frequency of ESBL production among Enterobacteriaceae $(n=92)$

Out of 92 Enterobacteriaceae 53 (57.6\%) were showing multidrug resistance (MDR). ${ }^{13}$ These were subjected for ESBL detection by phenotypic disc diffusion test. Among these 36(38.8\%) were confirmed ESBL producers. Highest prevalence (75.0\%) was shown by strains of E. Colifollowed by Klebsiella spp. (16.6\%), Citrobacter spp., Proteus spp and Providencia spp. (2.7\%) as shown in Fig. 5

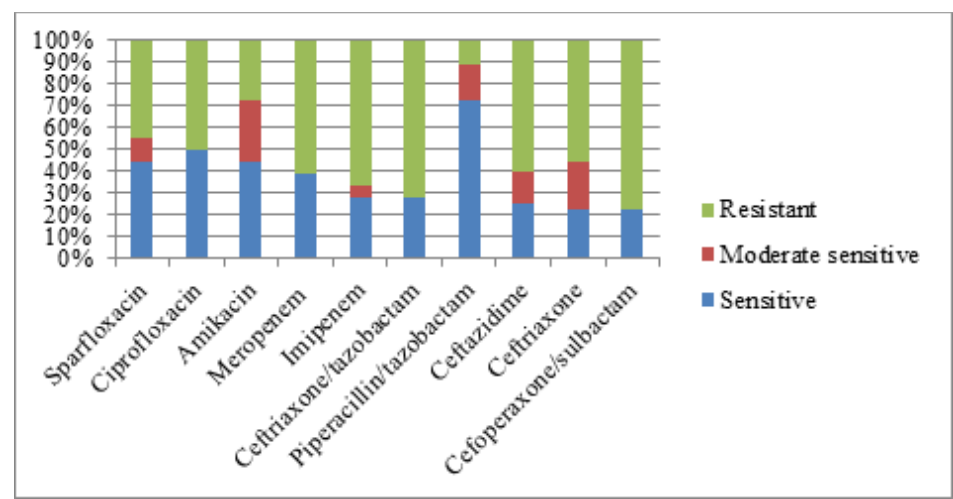

Fig. 6: Antibiotic sensitivity pattern for pseudomonas $\operatorname{spp}(\mathrm{n}=18)$

Pseudomonas spp. was the second most common isolated organism, it showed maximum sensitivity $72.2 \%$ to piperacillin/ tazobactam and maximum resistance $77.7 \%$ towards combination of cefoperzone/ sulbactam. Antibiotic sensitivity pattern of Pseudomonas spp. is given Fig. 6

The third most common isolated organism was Enterococci spp. showed maximum sensitivity towards nitrofurantoin $(96.6 \%)$, amikacin $(81.2 \%)$, gentamicin $(75.0 \%)$ and maximum resistance to fluroquinolones $(50 \%)$.

\section{Discussion}

UTI ranks among the most common infection in developing countries $(47 \%-55 \%)^{4,16-18}$ and in the present study it was $51.12 \%$.

Our study showed a high prevalence of UTI in females $(73.57 \%)$ than in males $(35.14 \%)$ which correlates with other findings which also revealed that the frequency of UTI is greater in females as compared to males. ${ }^{4,5,14,15}$ The reason behind this high prevalence of UTI in females may be due to close proximity of the urethral meatus to the anus, shorter urethra, sexual intercourse favouring the entry of bacteria into urethra and other common reasons are incontinence, and pregnancy., ${ }^{4,5,14}$ Females of the age group 15-49 years were found to be more susceptible $(90.69 \%)$ to UTI followed by 65-90 years (82.93\%). These findings are similar to other reports which showed that females are more prone to UTIs than males during adolescence and old age. ${ }^{15-17}$ While increasing incidence of UTI in young age females are associated with high sexual activity, recent use of a diaphragm with spermicide, and a history of recurrent UTIs, ${ }^{16,17}$ in elderly female it is due to less vaginal acidity and waxing defense mechanism.

In the present study, diabetes mellitus (33.08\%) was the most frequently associated risk factor for UTI 
which corroborates with the incidence of UTI as reported by Rawal et al (42.9\%) inAhmedabad. ${ }^{17}$ Diabetes mellitus is associated with many complications and in the long run it has some major effects on the urinary system which makes diabetic patients more vulnerable to UTI. ${ }^{18}$ Hyperglycemic urine promotes rapid bacterial growth and colonization. Autonomic neuropathy in diabetes mellitus impairs bladder emptying and subsequent urological manipulation pre-dispose to UTI. ${ }^{18}$ Pregnancy also showed high association with UTI (23.9\%) among females. Manjula et al in Bangalore reported $49.9 \%$ incidence of UTI in their study. ${ }^{19}$ The hormonal changes in pregnancy leads to decreased bladder tone, diminished peristalsis and dilatation of renal pelvis and ureter. Pregnancy produces physical obstruction in the female urinary tract, leading to retention of urine which is one of the important risk factor for the development of the infection. ${ }^{20,21}$ Catheterization was also a common risk factor in the present study leading to catheterassociated UTI in any age group. For either short- or long-term catheters, the infection rate is $8.08 \%$. Infection spreads by biofilm formation on both internal (intraluminal route) and external (periurethral route) catheter surface. ${ }^{19,22,23}$

The uropathogens isolated were similar to many other studies from different regions of India. Our study corroborates with the studies done by Bhargavi $e a^{24}$ in southeast India, Murugan et $a l^{25}$ in Tamil Nadu and Prakash $e a^{26}$ in U.P.

Gram-negative organisms are the most common organisms causing UTIs, and they collectively account for more than $75 \%$ of cases. Higher incidence of Gram negative bacteria belonging to Enterobacteriaceae, in causing UTI is associated with many factors which are responsible for their attachment to the uroepithelium. In addition, they are able to colonize in the urogenital mucosa with adhesins, pili, fimbriae, and P-1 blood group phenotype receptor. ${ }^{19}$

In our study Escherichia coli was the most common uropathogen accounting for $47.05 \%$ of cases. The findings are similar to studies done by Lakshmiet $a l^{28}(66.45 \%)$ and Raval et $a l^{17}(35 \%)$. The incidence of $E$. coli as a causative pathogen of UTI in India varies from $48 \%$ to $65 \%$ as reported by various studies done earlier. $^{18-22}$

Pseudomonas spp. was the second most common uropathogen accounting for $13.2 \%$ of cases which was similar to study done by Prakash et al $(12.90 \%) .{ }^{26}$ In our study only $11.7 \%$ of the isolates were Enterococci spp. According to study of Sood et al. from Jaipur Enterococci spp. accounted for $9.24 \%$ of urinary tract isolates. $^{15}$

The antibiotic sensitivity pattern was similar to other studies done in India. ${ }^{12,24,28,29}$ The members of Enetrobacteriaceae were showing maximum sensitivity towards nitrofurantoin (95.5\%), amikacin(75.5\%), gentamicin $(65.5 \%)$ and maximum resistance towards norfloxacin $(82.6 \%)$, nalidixic acid(76.0\%) which correlates with the study results of Aruna et $a l^{33}$ and Eshwarappa et $a l^{32}$ and resistance towards third generation cephalosporin like ceftazidime (37.7\%), ceftriaxone $(27.7 \%)$ and cefotaxime $(23.3 \%)$ were lower as compared to studies done by Gupta et al. ${ }^{29}$ The sensitivity pattern for third generation cephalosporin reported by Gupta et $a l^{29}$ was ceftazidime $48.3 \%$, ceftriaxone $33.7 \%$ and cefotaxime $39.4 \%$. Resistance towards cefotaxime $(70 \%)$ ceftriaxone $(68.8 \%)$ and ceftazidime $(62.2 \%)$ was analogous to studies by Bhargavi et $a l^{24}$ (cefotaxime $69 \%$, ceftazidime $65 \%$, ceftriaxone $60 \%$ ) and Manjunath et $a l^{30}$ (cefotaxime $68 \%$, ceftriaxone $65 \%$, ceftazidime $63 \%$ ).

Pseudomonas spp was sensitive to piperacillin/tazobactam is $72.2 \%$, ciprofloxacin $50 \%$, for amikacin and sparfloxacin $44.4 \%$, meropenem $38 \%$, for both imipenem and ceftazidime $27.7 \%$, for both ceftriaxone and cefoperazone/sulbactam $22.2 \%$. It was resistant to cefoperazone/sulbactam $77.7 \%$, ceftriaxone/tazobactam $72.2 \%$, resistance for both imipenem and ceftazidime was $66 \%$. Sensitivity and resistance pattern were similar to studies done by Sood et $a l^{15}$ in Jaipur and Kalal et $a l^{36}$ in Bangalore.

The antibiotic sensitivity pattern for third highest recorded organism Enterococcous spp. was as follows: (96.6\%) sensitive for nitrofurantoin, (81.2\%) for amikacin, (75\%) for gentamicin, $(62.5 \%)$ for nalidixic acid, (50\%) for both norfloxacin and gatifloxacin. Sensitivity pattern was similar to studies by Sood $e t a l^{15}$ in Jaipur and Llyods et $a l^{27}$ in South Michigan.

In our study most of Enterobacteriaceae were showing multi-drug resistance and which were subjected for ESBL detection. Out of 53(57.6\%) isolates were tested for ESBL production and $36(67.9 \%)$ were confirmed for ESBL production and findings corroborates with previous reports from Bangalore ${ }^{32}$ and Mumbai. ${ }^{33}$

Extended spectrum $\beta$ - Lactamase producers do not respond to the usually prescribed empirical therapy and there is an increased risk of associated morbidity and mortality. Factors which might have led to the high prevalence of the ESBL producers could be indwelling catheters, invasive procedures, and severity of the illness and excessive use of cephalosporins. Presently, alternative antimicrobial therapy to treat ESBL-positive UTI on outpatient basis is limited. Carbapenems are the most effective drugs in this situation ${ }^{34,35}$ but need to be administered intravenously or intramuscularly. All this and the high rate of ESBL positivity in the present study warrant a change in the empirical therapy of UTI to prevent the complications.

\section{Conclusion}

Escherichia. coli was the most common uropathogen isolated in our hospital, and is showing highest sensitivity towards nitrofurantoin and resistant towards norfloxacin and third generation 
cephalosporins. Extended spectrum beta- lactamase producers were also reported. Diabetic patients were at a higher risk of developing UTI. Higher prevalence of UTI was seen in females.

\section{References}

1. Flores-Mireles, AL; Walker, JN; Caparon, M; Hultgren, SJ, "Urinary tract infections: epidemiology, mechanisms of infection and treatment options.". Nature Reviews. Microbiology.(2015) 13 (5):269-84.

2. Gulati, S., Kher, V., Gupta, A., Arora, P., Rai, P.K. and Sharma, R.K, "Spectrum of Infections in Indian Children with Nephrotic Syndrome. Pediatric Nephrology"(1995)9,431-34.

3. Szasz M, Lehotkai N, Kristóf K, Szabó D, Nagy K, "Prevalence and antimicrobial resistance of uropathogens in different inpatient wards" Acta Microbiol Immunol Hung. (2009) 56:375-87.

4. Colgan, R; Williams, "Diagnosis and treatment of acute uncomplicated cystitis" American family physician (2011) 84 (7):771-6.

5. Kunin, C.M., "Urinary Tract Infections in Females". Clinical Infectious Diseases, (1994)18:1-12.

6. Kunin, C.M. "Detection, Prevention and Management of UTIs. 5th Edition”, Lea \&Febiger, (1997) Philadelphia.

7. Kashef N, Djavid GE, Shahbazi S., "Antimicrobial susceptibility patterns of community-acquired uropathogens in Tehran”, Iran. J Infect Dev Ctries. 2010 (4)202-6.

8. Baron EJ, Peterson LR, Finegold SM. 12th ed. St Louis: Mosby; 2007. Bailey and Scott's Diagnostic Microbiology; pp. 216-47.

9. Win, W.C., Stephen, D.A., Stephen, A., Janda, W., Koneman, E.W., Schrehrenberg, P. 2005. Koneman's Color Atlas and Textbook of Diagnostic Microbiology, 6th ed., Lipincott, William and Wilkins.

10. Nadagir, S.D., Chunchanur, S.K., Halesh, L.H., Yasmeen, K., Chandrashakhar, M.R., Patil, B.S. "Significance of Isolation And Drug Susceptibility Testing Of Non-Candida Albicans Species Causing Oropharyngeal Candidiasis In HIV Patients". Southeast Asian J Trop Med Public Health (2008) 39: 492-5.

11. Clinical and Laboratory Standards Institute. Performance standards for antimicrobial susceptibility testing; (2017).27thinformational supplement, Wayne, PA, USA. 2017; 32 (3) M100-S27.

12. CLSI. Performance standards for antimicrobial disc susceptibility tests; approved standard - 11th ed., CLSI 2012; document M02-A11.

13. MagiorakosAP, SrinivasanA, CareyRB,et al. "Multidrug-resistant, extensively drug-resistant and pandrug-resistant bacteria: an international expert proposal for interim standard definitions for acquired resistance", ClinMicrobiol Infect, (2012) (18)268-81.

14. F. A. Orrett, "Urinary tract infections in general practice in a rural community in South Trinidad," Saudi Medical Journal, (2001) (22)537-40.

15. S. Sood and R. Gupta, "Antibiotic resistance pattern of community acquired uropathogens at a tertiary care hospital in Jaipur, Rajasthan," Indian Journal of Community Medicine, (2012) (37)39-44.

16. Devanand Prakash and Ramchandra Sahai Saxena, "Distribution and Antimicrobial SusceptibilityPattern of Bacterial Pathogens Causing Urinary Tract Infection in Urban Community of Meerut City, India," ISRN Microbiology,(2013)30-43.
17. Raval, R., Verma, R.J. and Kareliya, H, "ClinoPathological Features of Urinary Tract Infection in Rural India. Advances in Infectious Diseases" (2015)5,132-9.

18. Ramana BV, Chaudhury A, "Prevalence of uropathogens in diabetic patients and their resistance pattern at a tertiary care centre in south India”. Int J Biol Med Res. (2012),3,1433.

19. Manjula N G, Math G C, Patil S A, Gaddad S M, Shivannavar C T, "Incidence of urinary tract infections and its aetiological agents among pregnant women in Karnataka Region" Journal of Advance Microbiogy. (2013),3,473-8.

20. MarahattaR,Dhungel BA, Pradhan P, Rai SK, Choudhury DR, “Asymptomatic Bacteriuria among pregnant women visiting Nepal medical college teaching hospital, Kathmandu, Nepal" Nepal Med Coll J, 2011(2), 107-110.

21. Lavanya $\mathrm{S}$ V, Jogalakshmi D, “Asymptomatic bacteriuria in antenatal women. Indian Journal of Medical Microbiolgy", (2002) 20,105-6.

22. I. Shaifali, U. Gupta, S. E. Mahmood, and J. Ahmed, "Antibiotic susceptibility patterns of urinary pathogens in female outpatients," North American Journal of Medical Sciences, (2012), 4,163-169.

23. R. N. Das, T. S. Chandrashekhar, H. S. Joshi, M. Gurung, N. Shrestha, and P. G. Shivananda, "Frequency and susceptibility profile of pathogens causing urinary tract infections at a tertiary care hospital in western Nepal," Singapore Medical Journal, (2006) 47,281-5.

24. Bhargavi PS, GopalaRao TV, Mukkanti K, Dinesh Kumar B, Krishna TP, "Increasing emergence of antibacterial resistance mainly in uropathogens:southeast part of India”. Intl J Microbiol Res 2010,2,1-6.

25. Murugan K, Savitha T, Vasanth S, "Retrospective study of antibiotic resistance among uropathogens from rural teaching hospital, Tamilnadu, India”. Asian Pac J Trop Dis. (2012), 2,375-80.

26. Prakash D, Saxena RS. Distribution and Antimicrobial Susceptibility Pattern of Bacterial Pathogens Causing Urinary Tract Infection in Urban Community of Meerut City,India. ISRNMicrobiology. (2013),6,56-78.

27. Lloyds S, Zrvas M, Mahayni R, Lundstrom T, "Risk factors for enterococal urinary tract infection and colonization in a rehabilitation facility" Am J Infect Control (1998)26,35-9.

28. P. Lakshmi Vasantha* and K. SaiLeela, "Antibiotic Susceptibility Pattern of Uropathogens Isolated in a RuralTeaching Hospital in South India" .Int.J.Curr.Microbiol.App.Sci(2015) 4(6):160-7.

29. Gupta N, Kundra S, Sharma A, GautamV,Arora DR. Antimicrobial susceptibility of uropathogens in India. J Infect Dis AntimicrobAgents (2007)24:13-8.

30. Manjunath GN, Prakash R, Annam V, Shetty K, "Changing trends in the spectrum of antimicrobial drug resistance pattern of uropathogens isolated from hospitals and community patients with urinary tract infections in Tumkur and Bangalore”. Int J Biol Med Res (2011)2:504-7.

31. Kalal BS, Nagaraj S, "Urinary tract infections: A retrospective, descriptive study of causative organisms and antimicrobial pattern of samples received for culture, from a tertiary care setting". Germs. (2016),4,132-8.

32. Eshwarappa M, Dosegowda R, Aprameya I V, Khan M W, Kumar P S, Kempegowda P, “Clinico- 
microbiological profile of urinary tract infection in South India”. Indian J Nephrol (2011),21,30-6.

33. Aruna K, Mobashshera T, "Prevalence of extended spectrum beta-lactamase production among uropathogens in south Mumbai and its antibiogram pattern”. EXCLI Journal. (2012),11,363-72.

34. . DeBusscher J, Zhang L, Buxton M, Foxman B, Barbosa-CesnikC, "Persistent extended-spectrum betalactamase urinary tract infection" Emerg Infect Dis. (2009), 15:1862-4.

35. AnupSaha, TapanMajumdar, ArunabhaDasgupta and PurnimaSaumandal. Prevalence of extended spectrum beta-lactamases (ESBLs) among uropathogenes at a tertiary care hospital in Tripura.The Health Agenda,(2015), 3. 215.

How to cite this article: Kaushik C, Gangadhar NK, Subrahmanya BK, Kotigadde S. Anti-biogram pattern of uro-pathogens isolated from patients in a Tertiary Care Hospital in Karnataka, India. Indian $\mathbf{J}$ Microbiol Res 2018;5(1):24-30. 